\title{
Pyrrolidinomethyl tetracycline in cavernous sinus thrombosis
}

\author{
S. R. K. MALIK, A. K. GUPTA, GURBAX SINGH, AND \\ S. CHOUDHRY
}

From the Department of Ophthalmology, Maulana Azad Medical College, New Delhi, India

Before the advent of antibiotics, cavernous sinus thrombosis of septic origin was invariably fatal (Grove, I936). After the introduction of sulphonamides and antibiotics, Shaw (1952) collected from the literature sixty cases of cavernous sinus thrombosis in which these drugs were used, and observed that the mortality rate had come down to I $1 \cdot 7$ per cent., but that 77 per cent. of those who survived showed residual abnormalities. Clune (1963) reported a survival rate of 72 per cent. with a 50 per cent. morbidity rate in those who recovered. In an attempt to reduce the high morbidity rate in survivors, newer antibiotics and anti-inflammatory and anti-thrombotic agents have been tried in various combinations. The present study reports our experience of treating seven cases of septic cavernous sinus thrombosis with pyrrolidinomethyl tetracycline (Reverin).

This is a pyrrolidinomethyl derivative of tetracycline with the molecular formula $\mathrm{C}_{27} \mathrm{H}_{33} \mathrm{O}_{8} \mathrm{~N}_{3}$. It is a water-soluble compound which can be administered intravenously or intramuscularly; it quickly attains high and reliable blood concentrations and the normal intestinal flora are not much affected. After the intravenous injection of $275 \mathrm{mg}$. Reverin the initial serum concentrations are almost ten times as high as after the oral administration of $250 \mathrm{mg}$. tetracycline hydrochloride (Strauch and Koch, I958); 8 hours later the former is still almost twice as high as the latter, and even after 24 hours therapeutic blood levels of Reverin can still be demonstrated (Finland, Purcell, Wright, Del Love, Mou, and Kass, 1954). Dimmling, Huner, Lutzeyer, and Simon (1958) have shown that the potency and bacteriostatic range of Reverin is similar to that of tetracycline hydrochloride, but with a much lower general and local toxicity.

Because of these advantages, it has been extensively used in fulminating infections with encouraging results, and it is therefore surprising that only one previous report of its use in a case of cavernous sinus thrombosis has been found (Khare, 1967).

The usual organisms encountered in cases of cavernous sinus thrombosis are the Streptococcus, Staphylococcus, B. proteus, B. pyocyaneus, Pneumococcus, and Meningococcus (Walsh, 1957). Tempea and Gorun (1959) stated that the first two organisms were most commonly encountered, and Clune ( 1963 ) found Staphylococcus aureus (coagulase-positive) to be the most frequent.

Reverin is effective against all these organisms (Finland and others, I954), but sulphonamides and antibiotics, including broad-spectrum tetracycline and chloramphenicol, have not proved to be very effective (Table III, p. I I5).

\section{Material and methods}

Seven cases of septic cavernous sinus thrombosis were treated with intravenous pyrrolidinomethyl 
tetracycline (Reverin) $275 \mathrm{mg}$. 8-hrly. Other supportive therapy, including anti-coagulants and Diamox, was added when necessary.

All the patients presented in a semiconscious or unconscious state, with high fever and neck rigidity. The clinical particulars are given in Table $\mathrm{I}$.

Table I Clinical particulars of seven cases of cavernous sinus thrombosis treated with intravenous Reverin

\begin{tabular}{|c|c|c|c|c|c|c|c|c|c|c|c|}
\hline \multirow[b]{2}{*}{$\begin{array}{l}\text { Case } \\
\text { no. }\end{array}$} & \multirow[b]{2}{*}{$\begin{array}{l}\text { Age } \\
\text { (yrs) }\end{array}$} & \multirow[b]{2}{*}{ Sex } & \multirow[b]{2}{*}{$\begin{array}{l}\text { Precipitating } \\
\text { cause }\end{array}$} & \multirow[b]{2}{*}{$\begin{array}{l}\text { Latent } \\
\text { period } \\
\text { (days) }\end{array}$} & \multicolumn{5}{|c|}{ General examination } & \multirow[b]{2}{*}{$\begin{array}{l}\text { Duration } \\
\text { of } \\
\text { treatment } \\
\text { (days) }\end{array}$} & \multirow[b]{2}{*}{ Outcome } \\
\hline & & & & & Appearance & $\begin{array}{l}\text { Blood } \\
\text { pressure }\end{array}$ & $\begin{array}{l}\text { Tempera- } \\
\text { ture } \\
\left({ }^{\circ} \mathrm{F} .\right)\end{array}$ & $\begin{array}{l}\text { Leucocyte } \\
\text { count } \\
\text { (per cu. } \\
\text { mm.) }\end{array}$ & $\begin{array}{l}\text { Erythrocyle } \\
\text { sedimentation } \\
\text { rate } \\
(\mathrm{mm} . / \mathrm{rst} \mathrm{hr})\end{array}$ & & \\
\hline I & 40 & $\mathbf{M}$ & Boil on nose & 8 & $\begin{array}{l}\text { Dehydrated } \\
\text { and toxic }\end{array}$ & $112 / 70$ & $103^{\circ}$ & 22,000 & 80 & 7 & Died \\
\hline 2 & 35 & $\mathbf{F}$ & $\begin{array}{l}\text { Septic } \\
\text { abortion }\end{array}$ & 4 & Unconscious & $100 / 60$ & $102^{\circ}$ & 10,000 & 60 & 15 & Gured \\
\hline 3 & 20 & $\mathbf{F}$ & $\begin{array}{l}\text { Furuncle on } \\
\text { nose }\end{array}$ & 5 & Semiconscious & $100 / 70$ & $102^{\circ}$ & 10,500 & 101 & 30 & Gured \\
\hline 4 & 40 & $\mathbf{F}$ & $\begin{array}{l}\text { Acute } \\
\text { exacerbation } \\
\text { of chronic } \\
\text { suppurative } \\
\text { otitis media }\end{array}$ & 8 & Semiconscious & $150 / 70$ & $103^{\circ}$ & I 9,200 & 80 & 20 & Cured \\
\hline 5 & 30 & $\mathbf{F}$ & $\begin{array}{l}\text { Septic } \\
\text { abortion }\end{array}$ & 9 & $\begin{array}{l}\text { Unconscious } \\
\text { Flexor } \\
\text { plantar } \\
\text { reflexes }\end{array}$ & $140 / 95$ & $104^{\circ}$ & 20,000 & 100 & 5 & $\begin{array}{l}\text { Died } \\
\text { 음 } \\
\end{array}$ \\
\hline 6 & 20 & $\mathbf{F}$ & $\begin{array}{l}\text { Abscess } \\
\text { on right } \\
\text { temple }\end{array}$ & 7 & Semiconscious & $140 / 80$ & $104^{\circ}$ & 12,000 & 100 & 24 & Cured $\frac{5}{\sqrt{2}}$ \\
\hline 7 & 12 & $\mathbf{M}$ & Not known & $\begin{array}{l}\text { Not } \\
\text { known }\end{array}$ & Semiconscious & I Io/8o & $103^{\circ}$ & 15,400 & 104 & 16 & Cured \\
\hline
\end{tabular}

The results of the ophthalmological examination are shown in Table II (opposite). All the patients showed lid oedema and conjunctival chemosis.

\section{Observations}

Of the seven patients treated, five $(7 \mathrm{I} \cdot 4$ per cent.) recovered without any residual effects, and two died with cardiorespiratory failure during the acute phase of the illness. The duration of treatment required in the survivors ranged from 15 to 30 days.

Unfortunately the blood cultures taken in our Cases 2, 3, and 4 were sterile. In one case Staphylococcus saprophyticus was grown.

\section{Comment}

The most significant feature noted in the present series was that there was no evidence of residual damage in the five patients who recovered, whereas previous reports have shown morbidity rates of from $5^{0}$ to 77 per cent. (Table III, opposite).

We therefore feel that pyrrolidinomethyl tetracycline is the drug of choice in the manage- $\stackrel{\oplus}{+}$ ment of septic cavernous sinus thrombosis, and that it should be administered from the start, without first trying less potent agents. 
Table II Ophthalmological findings in seven patients

\begin{tabular}{|c|c|c|c|}
\hline $\begin{array}{l}\text { Case } \\
\text { no. }\end{array}$ & Eye involved & Fundus & Other observations \\
\hline I & $\begin{array}{l}\text { Both (left more } \\
\text { than right) }\end{array}$ & $\begin{array}{l}\text { Hyperaemic disc } \\
\text { Dilated vessels }\end{array}$ & $\begin{array}{l}\text { Proptosis } \\
\text { Limitation of movement } \\
\text { Mastoid oedema }\end{array}$ \\
\hline 2 & Right & $\begin{array}{l}\text { Hyperaemic disc } \\
\text { Blurred margins } \\
\text { Dilated vessels }\end{array}$ & $\begin{array}{l}\text { Axial proptosis } \\
\text { Mastoid and facial oedema } \\
\text { Pupils semidilated with sluggish reaction }\end{array}$ \\
\hline 3 & $\begin{array}{l}\text { Both (left more } \\
\text { than right) }\end{array}$ & Not visible & $\begin{array}{l}\text { Axial proptosis } \\
\text { Exposure keratitis } \\
\text { Mastoid oedema }\end{array}$ \\
\hline 4 & $\begin{array}{l}\text { Both (left more } \\
\text { than right) }\end{array}$ & Normal & $\begin{array}{l}\text { Proptosis } \\
\text { Limitation of movement } \\
\text { Mastoid oedema }\end{array}$ \\
\hline 5 & Right & Hyperaemic disc & $\begin{array}{l}\text { Proptosis } \\
\text { Limitation of movement } \\
\text { Mastoid oedema } \\
\text { Pupils semidilated with sluggish reaction }\end{array}$ \\
\hline 6 & Right & Dilated vessels & $\begin{array}{l}\text { Proptosis } \\
\text { Limitation of movement }\end{array}$ \\
\hline 7 & Left & $\begin{array}{l}\text { Hyperaemic disc } \\
\text { Retinal oedema } \\
\text { Dilated vessels }\end{array}$ & $\begin{array}{l}\text { Proptosis } \\
\text { Limitation of movement }\end{array}$ \\
\hline
\end{tabular}

Table III Mortality and morbidity rates in cases reported by other workers compared with the seven cases here reported

\begin{tabular}{lrrll}
\hline Author & Year & $\begin{array}{c}\text { Total } \\
\text { cases }\end{array}$ & $\begin{array}{l}\text { Mortality } \\
\text { (per cent. })\end{array}$ & $\begin{array}{c}\text { Morbidity } \\
(\text { per cent. })\end{array}$ \\
\hline Shaw & I 954 & 60 & I I $\cdot 7$ & $77 \cdot 0$ \\
Yarington & I96 I & 878 & $80 \cdot 0$ & $75 \cdot 0$ \\
Clune & 1963 & 36 & $28 \cdot 0$ & $50 \cdot 0$ \\
& & 5 & $60 \cdot 0$ & $50 \cdot 0$ \\
\hline Present series & I969 & 7 & $28 \cdot 6$ & Nil \\
& & & & \\
\hline
\end{tabular}

\section{Summary}

Pyrrolidinomethyl tetracycline (Reverin) was given intravenously in seven cases of septic cavernous sinus thrombosis, and was found to be useful in eliminating residual morbidity in the five patients who recovered.

\section{References}

Clune, J. P. (1963) Amer. F. Ophthal., 56, 33

DimMLing, T., hUNER, H., LUTZEYER, w., and simon, G. (1958) Münch. med. Wschr., roo, 676 
Finland, M., PURGell, E. M., Wright, s. s., Del love, B., MoU, T. w., and Kass, E. H. (i954) J. Amer. med. Ass., I54, 56 I

Grove, w. E. (1936) Arch. Otolaryng. (Chicago), 24, 29

KHARE, B. B. (I967) Brit. 7. Ophthal., 51, 7 I 2

SHAw, R. E. (1952) Brit. J. Surg., 40, 40

STRAuch, D., and косн, E. (1958) Münch. med. Wschr., roo, 668

TEMPeA, v., and gorun, G. (1959) Arch. Otolaryng. (Chicago), 69, 220

WAlsh, F. B. (1957) “Clinical Neuro-Ophthalmology”, 2nd ed., p. 8o8. Williams and Wilkins,

Baltimore

yarington, c. T. (1961) Ann. Otol. (St. Louis), 70, 263 